\title{
QCD analysis of $D^{*}$-meson fragmentation functions and their uncertainties at NNLO *
}

\section{Maryam Soleymaninia}

Department of Physics, Shahid Rajaee Teacher Training University, Lavizan, Tehran 16788, Iran School of Particles and Accelerators, Institute for Research in Fundamental Sciences (IPM), P.O.Box 19395-5531, Tehran, Iran

E-mail: Maryam_Soleymaninia@ipm.ir

\section{S. Mohammad Moosavi Nejad}

Faculty of Physics, Yazd University, P.O.Box 89195-741, Yazd, Iran

School of Particles and Accelerators, Institute for Research in Fundamental Sciences (IPM),

P.O.Box 19395-5531, Tehran, Iran

E-mail: Mmoosavi@yazd.ac.ir

In this paper, we present a set of charmed-meson $D^{*}$ at next-to-leading order (NLO) and, for the first time, at next-to-next-to-leading order (NNLO) accuracy. This new sets of charmed-meson $D^{*}$ FFs are obtained through global QCD fits to all relevant data sets from single-inclusive $e^{+} e^{-}$ annihilation. The "Hessian approach" are applied for the determination of FFs uncertainties as well as the corresponding observables. We compared the resulting $D^{*}$ FFs with the results in the litrature. The theory predition using the extracted FFs are also compared with the available data sets for single-inclusive $D^{* \pm}$-meson production in electron-positron annihilation.

XIIIth Quark Confinement and the Hadron Spectrum, 31 July 2018 to 6 August 2018 Maynooth University, IRLAND

* On behalf of SKM18 Collaboration 


\section{Introduction}

Studies over the past two decades have provided valuable vital information on the structure of hadrons. Fragmentation functions (FFs) $[1,2,3,4,5,6,7]$ describe the non-perturbative part of hard-scattering processes and along with the parton distribution functions (PDFs) of initial hadrons (in a given hadron-hadron collision) and parton-level differential cross sections are three important ingredients to obtain theoretical predictions for the hadroproduction cross sections [8, 9, 10, 11, 12].

The FFs are studying in hadrproduction processes such as electron-positron $\left(e^{+} e^{-}\right)$singleinclusive annihilation (SIA), lepton-hadron semi-inclusive deep inelastic scattering (SI-DIS) and hadron-hadron collisions. Theoretically the cleanest process to extract the fragmentation densities is SIA because we do not require the simultaneous knowledge of PDFs and FFs. Recently, different analysis have been focused on extracting of FFs for light and heavy hadrons at NLO and also NNLO accuracies in perturbative QCD (pQCD) $[2,5,6,13,14]$. The calculation of FFs at high order corrections NNLO of pQCD is possible just for electron-positron $e^{+} e^{-}$annihilation while the calculations for the hard processes in SI-DIS and $p p$ collisions at NNLO are not accessible yet.

In this paper, for the first time, a comprehensive QCD analysis is performed to obtain a set of charm- and bottom-quarks into $D^{* \pm}$-mesons and their uncertainties at NNLO through a global QCD fit to SIA data from ALEPH [15] and OPAL [16] collaborations at LEP. Our analysis is entitled as "SKM18" and using the zero-mass variable flavor scheme (ZM-VFN). It is limited to SIA data only cross sections at NLO and NNLO accuracies.

In Ref [17], the authors have determined non-perturbative charmed-meson FFs at NLO in the general-mass variable flavor number scheme (GM-VFNs), using the SIA data from Belle, CLEO, ALEPH and OPAL Collaborations. Recently, the authors of Ref. [13] have determined the FFs of charged $D^{*}$-meson at NLO accuracy using the available data sets for SIA $D^{*}$-meson and in protonproton scattering in which the gluon FF can be well constrained via proton-proton scattering data.

This paper is organized as follows. In Sec. 2, the QCD analyses of hadronization process in electron-positron $e^{+} e^{-}$annihilation are discussed. In this section, we also describe our formalism and parametrizations for the charmed-meson $D^{* \pm}$ FFs at a given input scale as well as the analyzed experimental data sets. In Sec. 3, the results of our $D^{* \pm}$-FFs and their uncertainties are discussed and compared with other in literature. Our result and conclusion are summarized in Sec. 4.

\section{Theory settings and FFs parametrization}

According to the factorization theorem of improved QCD-parton model, the differential cross section of electron-positron $e^{+} e^{-}$annihilation can be written as a convolutions of perturbative part of partonic cross sections, $d \sigma_{i}\left(x_{i}, \mu_{R}, \mu_{F}\right) / d x_{i}$, with the non-perterbative part of $D_{i}^{D^{*}}\left(z, \mu_{F}^{2}\right)$-FFs, where $i$ stands for one of the flavors of fragmenting partons; $i=g, u, \bar{u}, \cdots, b, \bar{b}$. This convolution reads

$$
\begin{aligned}
& \frac{1}{\sigma_{\mathrm{t} o t}} \frac{d}{d x_{D}} \sigma\left(e^{+} e^{-} \rightarrow D^{*} X\right)= \\
& \sum_{i} \int_{x_{D}}^{1} \frac{d x_{i}}{x_{i}} D_{i}^{D^{*}}\left(\frac{x_{D}}{x_{i}}, \mu_{F}\right) \frac{1}{\sigma_{\mathrm{t} o t}} \frac{d \sigma_{i}}{d x_{i}}\left(x_{i}, \mu_{R}, \mu_{F}\right) .
\end{aligned}
$$


The renormalization $\left(\mu_{R}\right)$ as well as factorization $\left(\mu_{F}\right)$ scales are made consists of setting $\mu^{2}=$ $\mu_{F}^{2}=Q^{2}$ in SKM1 8 analysis. The variable $x_{i}=2\left(p_{i} \cdot q\right) / q^{2}$, where $p_{i}$ is the four-momentum of parton $i$. The total cross section up to NNLO for $e^{+} e^{-}$annihilation into hadrons can be written as,

$$
\begin{aligned}
\sigma_{\mathrm{t} t}= & \frac{4 \pi \alpha^{2}(Q)}{Q^{2}}\left(\sum_{i}^{n_{f}} \tilde{e}_{i}^{2}(Q)\right)\left(1+\alpha_{s} K_{\mathrm{QCD}}^{(1)}\right. \\
& \left.+\alpha_{s}^{2} K_{\mathrm{QCD}}^{(2)}+\cdots\right)
\end{aligned}
$$

In order to choose the best parametrization for SKM1 8 global analysis of $D^{* \pm}$-FFs, we have tested different functional input. Finally we decided adopted the Bowler [18] parametrization form because one can control this parametrization considering the low number of data for $D^{* \pm}$-meson FFs. Therefore, we parameterize the $z$ distributions of the $c(\bar{c})$ as well as $b(\bar{b})$ quark FFs at their starting scales $\mu_{0}$ as suggested by Bowler. It is given by:

$$
D_{i}^{D^{* \pm}}\left(z, \mu_{0}^{2}\right)=N_{i} z^{-\left(1+\alpha_{i}^{2}\right)}(1-z)^{\beta_{i}} e^{-\alpha_{i}^{2} / z} .
$$

As one can see, this parametrization comes with three free parameters; $\mathrm{N}, \alpha$ and $\beta$. Our fitting procedure is going as follows. At the scale $\mu_{0}$, the FFs of gluon and light quarks $(q=u, d, s)$ are set to zero, i.e.

$$
D_{i}^{D^{* \pm}}\left(z, \mu_{0}^{2}\right)=0, \quad i=u, \bar{u}, d, \bar{d}, s, \bar{s}, g .
$$

Then, the light and gluon FFs are evolved to the higher scales $\mu^{2}>\mu_{0}^{2}$ using the DGLAP evolution equations [19] at NLO or NNLO accuracy.

ALEPH [15], OPAL [16], CLEO [20] and Belle [21] Collaborations are reported most of the experimental data for $D^{* \pm}$ in electron-positron $\left(e^{+} e^{-}\right)$annihilation. An overview of the data included in SKM1 8 global analysis of $D^{* \pm}$ FFs is presented in our previous analysis [14] for the total, $c$-tagged and $b$-tagged SIA cross sections from ALEPH [15] and OPAL [16] Collaborations. OPAL and ALEPH Collaborations at Large electron-positron (LEP) present their experimental data sets at $Q=M_{Z}$ which is the mass of the $Z$ boson, while other Collaborations such as Belle and CLEO provide their data in lower energy, i.e. $Q=10.5 \mathrm{GeV}$. In this range of energy, all $D^{* \pm}$ FFs in electron-positron $\left(e^{+} e^{-}\right)$annihilation coming from bottom decays are excluded because they are below the mass threshold of $b$-quark.

The determination of non-perturbative FFs through global QCD fits to the experimental data sets is a statistical procedure that necessarily implies a variety of assumptions. The most important one is the parameterization functions for the charmed-meson FFs at a given input scale as well as and the propagation of the experimental uncertainties into them $[2,3,4,22,23,24]$. The assessment of uncertainties of PDFs and also the corresponding observables have seen significant efforts in very recent QCD analyses (see for example [9, 10, 25, 26]). Among the different approaches in literature, the "Hessian method" [27, 28, 29] is the most reliable ones. The well-known and practical method "Hessian method", has been widely used to extract the uncertainties of the PDFs, polarized PDFs and nuclear PDFs as well as the corresponding observables in our previous analyses $[30,31,32,33,34,35,36]$. Since, the technical details of the Hessian approach are described in literature, we refer the readers to the mentioned references. 


\section{Results and discussions}

In the following section, we turn to our numerical results for the global analysis of $D^{* \pm}$-FFs from SIA data. The detailed discussions can be found in Ref. [14], then we will review some important findings. We present our NLO and NNLO FFs analyses for the charm and bottom densities in zero-mass variable flavor number scheme (ZM-VFNS).

The obtained $D^{* \pm}$ FFs and their uncertainties derived using Hessian approach are presented at the input scale $\mu_{0}^{2}=18.5 \mathrm{GeV}^{2}$ in Fig. 1 as well as for a higher values of $\mu^{2}=100 \mathrm{GeV}^{2}$ in Fig. 2. The results are correspond to the one- $\sigma$ uncertainty bands. Our results in Fig. 2 are compared to the central value of KKKS 08 analysis [17] as well as very recent analysis by AKSRV17 [13].
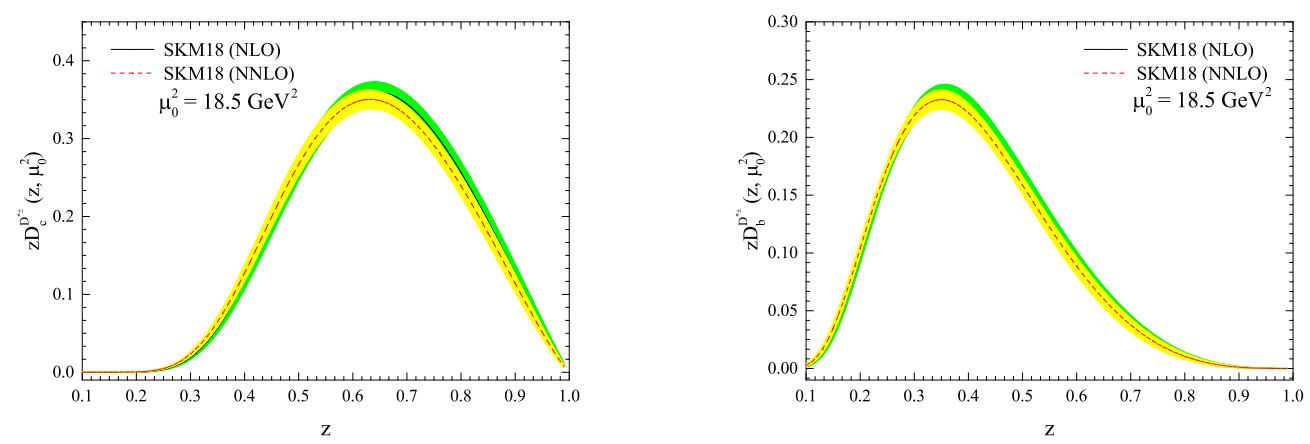

Figure 1: The obtained fragmentation densities and their uncertainties (shaded bands) are shown for the charmed-meson $z D_{i}^{D^{* \pm}}$ at the initial scale of $\mu_{0}^{2}=18.5 \mathrm{GeV}^{2}$ for $c$ and $b$ FFs both at NLO and NNLO accuracy.

As one can conclude from the presented results, the most difference between SKM1 8, KKKS 08 as well as the AKSRV1 7 analysis is in the extracted gluon FF. The charm and bottom FFs from SKM1 8 analysis are in agreement with KKKS 08 and AKSRV17, however the charm FF in KKKS 08 are slightly higher than other.

\section{Summary}

In this article, we have presented the non-perturbative FFs of partons into the $D^{* \pm}$-meson at NLO perturbative QCD as well as at NNLO. These sets of FFs have been obtained from global analyses of single-inclusive electron-positron $\left(e^{+} e^{-}\right)$annihilation data sets. In order to consider the heavy quark contributions, SKM1 8 analyses have been done in the ZM-VFN scheme in which all quarks are treated as massless partons. Our phenomenological analyses are significant in, at least, two major respects. Firstly, we applied all SIA experimental data sets in SKM1 8 NLO and NNLO QCD analyses. Secondly, for the first time, we considered the NNLO accuracy in SKM1 8 global fit. The obtained results show that SKM1 8 calculations at NNLO come with much smaller theoretical uncertainties relative to the NLO calculations which could reflects the stability of the NNLO analysis. In addition, we found that the experimental uncertainties for the $D^{* \pm}$-FFs and SIA 

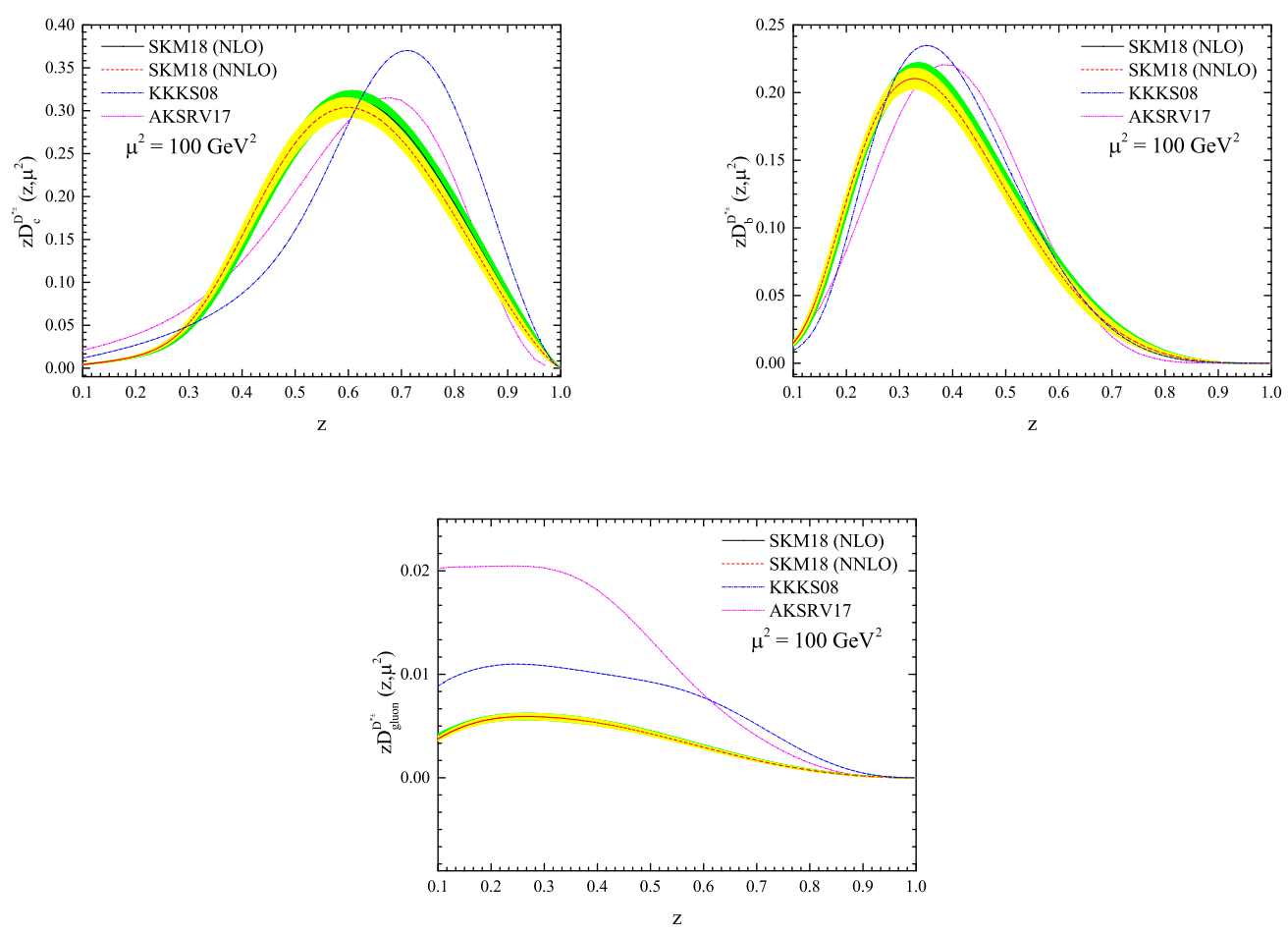

Figure 2: Fragmentation densities including their uncertainties (shaded bands) are shown for the charge meson $z D_{i}^{D^{* \pm}}$ at $\mu^{2}=100 \mathrm{GeV}^{2}$ for $c, b$ and gluon FFs both at NLO and NNLO accuracy. SKM1 8 results are also compared with the KKKS 08 analysis [17] as well as very recent analysis by AKSRV17 [13].

cross sections are similar in size both for the NLO and NNLO approximations. These findings are significantly in agreements with previous results reported in the literature. The theory prediction for the SIA cross section based on SKM1 $8 D^{* \pm}$-meson FFs are in good agreements with all data analyzed.

\section{Acknowledgments}

Authors would like to thank the School of Particles and Accelerators, Institute for Research in Fundamental Sciences (IPM) for financial support of this project. We are especially grateful Hamzeh Khanpour for many useful discussions and comments.

\section{References}

[1] V. Bertone et al. [NNPDF Collaboration], "Charged hadron fragmentation functions from collider data,” Eur. Phys. J. C 78, no. 8, 651 (2018), [arXiv:1807.03310 [hep-ph]].

[2] V. Bertone et al. [NNPDF Collaboration], "A determination of the fragmentation functions of pions, kaons, and protons with faithful uncertainties," Eur. Phys. J. C 77 (2017) no.8, 516 [arXiv:1706.07049 [hep-ph]]. 
[3] D. P. Anderle, F. Ringer and M. Stratmann, "Fragmentation Functions at Next-to-Next-to-Leading Order Accuracy,” Phys. Rev. D 92, no. 11, 114017 (2015) [arXiv:1510.05845 [hep-ph]].

[4] J. J. Ethier, N. Sato and W. Melnitchouk, "First simultaneous extraction of spin-dependent parton distributions and fragmentation functions from a global QCD analysis," Phys. Rev. Lett. 119, no. 13, 132001 (2017) [arXiv:1705.05889 [hep-ph]].

[5] M. Soleymaninia, M. Goharipour and H. Khanpour, "First global QCD analysis of charged hadron fragmentation functions and their uncertainties at next-to-next-to-leading order," Phys. Rev. D 98, 074002 (2018), [arXiv:1805.04847 [hep-ph]].

[6] A. Mohamaditabar, F. Taghavi-Shahri, H. Khanpour and M. Soleymaninia, "Determination of contributions from residual light charged hadrons to inclusive charged hadrons from $e^{+} e^{-}$ annihilation data," arXiv:1808.09255 [hep-ph].

[7] M. Soleymaninia, M. Goharipour and H. Khanpour, "Impact of unidentified light charged hadron data on the determination of pion fragmentation functions," Phys. Rev. D 99, no. 3, 034024 (2019), [arXiv:1901.01120 [hep-ph]].

[8] E. R. Nocera and M. Ubiali, “Constraining the gluon PDF at large x with LHC data,” PoS DIS 2017, 008 (2018), [arXiv:1709.09690 [hep-ph]].

[9] T. J. Hou et al., "CT14 Intrinsic Charm Parton Distribution Functions from CTEQ-TEA Global Analysis,” JHEP 1802, 059 (2018), [arXiv:1707.00657 [hep-ph]].

[10] K. J. Eskola, P. Paakkinen, H. Paukkunen and C. A. Salgado, "EPPS16: Nuclear parton distributions with LHC data," Eur. Phys. J. C 77, no. 3, 163 (2017) [arXiv:1612.05741 [hep-ph]].

[11] L. Frankfurt, V. Guzey and M. Strikman, "Dynamical model of antishadowing of the nuclear gluon distribution,” Phys. Rev. C 95, no. 5, 055208 (2017) [arXiv:1612.08273 [hep-ph]].

[12] M. Goharipour and H. Mehraban, "Study of isolated prompt photon production in $p$ - $\mathrm{Pb}$ collisions for the ALICE kinematics,” Phys. Rev. D 95, no. 5, 054002 (2017) [arXiv:1702.05738 [hep-ph]].

[13] D. P. Anderle, T. Kaufmann, M. Stratmann, F. Ringer and I. Vitev, "Using hadron-in-jet data in a global analysis of $D^{*}$ fragmentation functions,” Phys. Rev. D 96, no. 3, 034028 (2017) [arXiv:1706.09857 [hep-ph]].

[14] M. Soleymaninia, H. Khanpour and S. M. Moosavi Nejad, "First determination of $D^{*+}$-meson fragmentation functions and their uncertainties at next-to-next-to-leading order," Phys. Rev. D 97 , no. 7, 074014 (2018), [arXiv:1711.11344 [hep-ph]].

[15] R. Barate et al. [ALEPH Collaboration], "Study of charm production in Z decays," Eur. Phys. J. C 16, 597 (2000) [hep-ex/9909032].

[16] K. Ackerstaff et al. [OPAL Collaboration], "Measurement of $\mathrm{f}(\mathrm{c} \rightarrow \mathrm{D} *+\mathrm{X}), \mathrm{f}(\mathrm{b} \rightarrow \mathrm{D} *+\mathrm{X})$ and Gamma (c anti-c) / Gamma (hadronic) using D*+- mesons,” Eur. Phys. J. C 1, 439 (1998) [hep-ex/9708021].

[17] T. Kneesch, B. A. Kniehl, G. Kramer and I. Schienbein, "Charmed-meson fragmentation functions with finite-mass corrections,” Nucl. Phys. B 799, 34 (2008) [arXiv:0712.0481 [hep-ph]].

[18] M. G. Bowler, “e+ e- Production of Heavy Quarks in the String Model,” Z. Phys. C 11, 169 (1981).

[19] V. N. Gribov and L. N. Lipatov, Sov. J. Nucl. Phys. 15 (1972) 438 [Yad. Fiz. 15 (1972) 781]; G. Altarelli and G. Parisi, Nucl. Phys. B 126 (1977) 298; Sov. Phys. JETP 46 (1977) 641 [Zh. Eksp. Teor. Fiz. 73 (1977) 1216]. 
[20] M. Artuso et al. [CLEO Collaboration], "Charm meson spectra in $e^{+} e^{-}$annihilation at 10.5-GeV c.m.e.," Phys. Rev. D 70, 112001 (2004) [hep-ex/0402040].

[21] R. Seuster et al. [Belle Collaboration], "Charm hadrons from fragmentation and B decays in e+ eannihilation at $\mathrm{s}^{* *}(1 / 2)=10.6-\mathrm{GeV}$,” Phys. Rev. D 73, 032002 (2006) [hep-ex/0506068].

[22] A. Metz and A. Vossen, "Parton Fragmentation Functions," Prog. Part. Nucl. Phys. 91, 136 (2016) [arXiv:1607.02521 [hep-ex]].

[23] D. P. Anderle, T. Kaufmann, M. Stratmann and F. Ringer, "Fragmentation Functions Beyond Fixed Order Accuracy,” Phys. Rev. D 95, no. 5, 054003 (2017) [arXiv:1611.03371 [hep-ph]].

[24] D. de Florian, M. Epele, R. J. Hernandez-Pinto, R. Sassot and M. Stratmann, "Parton-to-Kaon Fragmentation Revisited,” Phys. Rev. D 95, no. 9, 094019 (2017) [arXiv:1702.06353 [hep-ph]].

[25] R. D. Ball et al. [NNPDF Collaboration], "Parton distributions from high-precision collider data," Eur. Phys. J. C 77, no. 10, 663 (2017) [arXiv:1706.00428 [hep-ph]].

[26] J. Gao, L. Harland-Lang and J. Rojo, “The Structure of the Proton in the LHC Precision Era," Phys. Rept. 742, 1 (2018), [arXiv:1709.04922 [hep-ph]].

[27] A. D. Martin, R. G. Roberts, W. J. Stirling and R. S. Thorne, "Uncertainties of predictions from parton distributions. 1: Experimental errors,” Eur. Phys. J. C 28, 455 (2003) [hep-ph/0211080].

[28] J. Pumplin, D. Stump, R. Brock, D. Casey, J. Huston, J. Kalk, H. L. Lai and W. K. Tung, “Uncertainties of predictions from parton distribution functions. 2. The Hessian method," Phys. Rev. D 65, 014013 (2001) [hep-ph/0101032].

[29] S. Shoeibi, H. Khanpour, F. Taghavi-Shahri and K. Javidan, "Determination of neutron fracture functions from a global QCD analysis of the leading neutron production at HERA," Phys. Rev. D 95, no. 7, 074011 (2017) [arXiv:1703.04369 [hep-ph]].

[30] S. Shoeibi, F. Taghavi-Shahri, H. Khanpour and K. Javidan, "Phenomenology of leading nucleon production in ep collisions at HERA in the framework of fracture functions," Phys. Rev. D 97, no. 7, 074013 (2018), [arXiv:1710.06329 [hep-ph]].

[31] F. Taghavi-Shahri, H. Khanpour, S. Atashbar Tehrani and Z. Alizadeh Yazdi, "Next-to-next-to-leading order QCD analysis of spin-dependent parton distribution functions and their uncertainties: Jacobi polynomials approach,” Phys. Rev. D 93, no. 11, 114024 (2016) [arXiv:1603.03157 [hep-ph]].

[32] H. Khanpour, S. T. Monfared and S. Atashbar Tehrani, "Study of spin-dependent structure functions of ${ }^{3} \mathrm{He}$ and ${ }^{3} \mathrm{H}$ at NNLO approximation and corresponding nuclear corrections," Phys. Rev. D 96, no. 7, 074037 (2017) [arXiv:1710.05747 [hep-ph]].

[33] H. Khanpour, S. T. Monfared and S. Atashbar Tehrani, "Nucleon spin structure functions at NNLO in the presence of target mass corrections and higher twist effects," Phys. Rev. D 95, no. 7, 074006 (2017) [arXiv:1703.09209 [hep-ph]].

[34] H. Khanpour and S. Atashbar Tehrani, "Global Analysis of Nuclear Parton Distribution Functions and Their Uncertainties at Next-to-Next-to-Leading Order,” Phys. Rev. D 93, no. 1, 014026 (2016) [arXiv:1601.00939 [hep-ph]].

[35] S. M. Moosavi Nejad, H. Khanpour, S. Atashbar Tehrani and M. Mahdavi, "QCD analysis of nucleon structure functions in deep-inelastic neutrino-nucleon scattering: Laplace transform and Jacobi polynomials approach,” Phys. Rev. C 94, no. 4, 045201 (2016) [arXiv:1609.05310 [hep-ph]].

[36] H. Khanpour, A. Mirjalili and S. Atashbar Tehrani, "Analytic derivation of the next-to-leading order proton structure function $F_{2}^{p}\left(x, Q^{2}\right)$ based on the Laplace transformation," Phys. Rev. C 95, no. 3 , 035201 (2017) [arXiv:1601.03508 [hep-ph]]. 